\title{
Invaginación intestinal en adultos como manifestación de enfermedad celíaca. Caso clínico
}

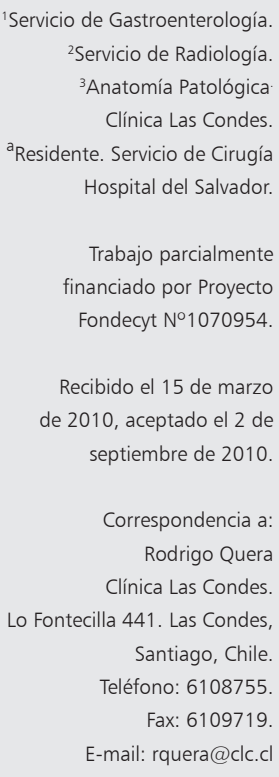

\author{
RODRIGO QUERA ${ }^{1}$, CLAUDIO HEINE T.a, \\ ANDRÉS O'BRIEN'², LUIS CONTRERAS ${ }^{3}$
}

\section{Celiac disease presenting as an intestinal intussusception. Report of one case}

\begin{abstract}
Acute abdominal pain caused by perforation, intestinal lymphoma or intussusception is an uncommon manifestation in adult celiac disease. We report a 49 yearold female with history of irritable bowel syndrome and osteoporosis consulting for acute abdominal pain and severe abdominal distention. Abdominal CT scan and magnetic resonance imaging showed a jejunal intussusception and other radiological alterations that suggested the possibility of celiac disease. Serological antibodies (endomysial and transglutaminase antibodies), endoscopy and the pathological study of duodenal biopsies confirmed the diagnosis. With a gluten free diet, the patient remains asymptomatic.
\end{abstract}

(Rev Med Chile 2010; 138: 1276-1280).

Key words: Celiac disease; Intussusception.
L a enfermedad celíaca (EC), una condición fuertemente asociada a los antígenos HLAtipo II DQ2 y DQ8 ${ }^{1}$, es definida como una enteropatía inmune provocada por una reacción al gluten ${ }^{2}$. Esta enfermedad de distribución global, puede ser diagnosticada a cualquier edad y afecta múltiples órganos y sistemas. Tiene una prevalencia mundial de alrededor de 1\%, sin embargo, se estima que ésta es aun mayor, ya que sólo 10\% de las personas afectadas son diagnosticadas ${ }^{3}$. En nuestro país no existen datos al respecto, pero es claro que su incidencia va en aumento, al igual que en el resto del mundo ${ }^{4}$.

La invaginación intestinal es un cuadro causado cuando un segmento del intestino (intususeptum) se introduce en el segmento distal adyacente del intestino (intususcipiens), siendo la causa más frecuente de obstrucción intestinal en niños $<5$ años. En estos casos, una lesión estructural del intestino es identificada solamente en el 5-15\% de los casos, y la mayoría de estos (> 80\%) se resuelve con un tratamiento conservador ${ }^{5}$. Por el contrario, en el adulto la invaginación intestinal es una patología infrecuente ya que representa sólo el 5\% de todos los casos de invaginación intestinal y $1-5 \%$ de las causas de obstrucción intestinal del adulto ${ }^{6}$. Estudios radiológicos recientes han demostrado que en pacientes adultos, sólo $30 \%$ de las invaginaciones intestinales presentan un punto de origen identificable y que sobre $50 \%$ de los pacientes sin este punto de origen identificable presentan una etiología idiopática ${ }^{7,8}$. Sin embargo, en su mayoría, estos casos no han sido seguidos de manera prolongada. Entre las posibles etiologías de las invaginaciones entero-entéricas sin un punto de origen destacan procesos inflamatorios (pancreatitis aguda, colecistitis, apendicitis), adherencias, enfermedad de Crohn, esclerodermia, fibrosis quística y EC 9 . Artículos, tanto en población pediátrica como adulta, han demostrado que la invaginación intestinal puede ser una forma de presentación de la $\mathrm{EC}^{10-14}$. Aunque ya existe 
Invaginación intestinal en enfermedad celíaca - R. Quera et al

un caso clínico a nivel nacional que muestra la invaginación intestinal como una manifestación de la $\mathrm{EC}^{13}$, creemos que nuestro caso confirma que esta etiología debe ser considerada en el diagnóstico diferencial de la invaginación intestinal sin un punto de origen, sobre todo si otros criterios radiológicos están presentes.

\section{Caso clínico}

Paciente de 49 años, sexo femenino, con antecedentes de síndrome intestino irritable tipo constipación desde la adolescencia, gastritis medicamentosa por uso de antiinflamatorios no esteroidales (AINE) el año 2000 (endoscopia digestiva alta duodeno normal), osteporosis el año 2004 y tiroiditis aguda el año 2005 en tratamiento con levotiroxina $75 \mathrm{mcg} / \mathrm{día}$. El año 2001 presentó cuadro de dolor abdominal en fosa iliaca derecha intenso, de inicio súbito, por lo que fue hospitalizada con diagnóstico de apendicitis aguda, la que se descartó por ecotomografía abdominal y una evolución clínica favorable. Dada la intensidad del cuadro clínico se realizó una colonoscopia que mostró la presencia de una compresión extrínseca a nivel cecal. Fue evaluada con TC de abdomen y pelvis donde se señaló que existía una masa en el anexo derecho que comprimía el ciego. Se decidió realizar una laparoscopia exploradora cuyo hallazgo fue la presencia de una distensión del colon derecho asociado a una profusa peristalsis del intestino delgado y del colon. Los ovarios eran normales y las trompas se apreciaban eritematosas y rígidas, por lo que se concluyó una salpingitis crónica bilateral, realizándose una salpingectomía bilateral. Las biopsias posteriores descartaron la presencia de cualquier proceso inflamatorio. $\mathrm{La}$ paciente se mantuvo en buenas condiciones generales presentando episodios aislados de malestar y distensión abdominal hasta agosto de 2009, fecha en que fue hospitalizada con diagnóstico probable de obstrucción intestinal por un nuevo cuadro de dolor abdominal agudo e intenso, asociado a distensión abdominal importante. Se realizó una TC de abdomen y pelvis donde destacaba la presencia de una lesión focal hepática sólida de un $\mathrm{cm}$ de diámetro en el segmento VI, no caracterizable en este estudio, además de múltiples áreas de invaginación en yeyuno, probablemente transitorias, no asociado a signos de obstrucción intestinal (Figura 1A -D). La paciente fue tratada en forma sintomática con buena respuesta y se dio de alta con indicación de estudio de resonancia magnética para evaluación de la lesión hepática. Este examen se realizó estando la paciente asintomática, describiéndose dos lesiones hepáticas de 3 y $10 \mathrm{~mm}$ en segmento IV y VI respectivamente, compatibles con hemangiomas, además de los siguientes ha-
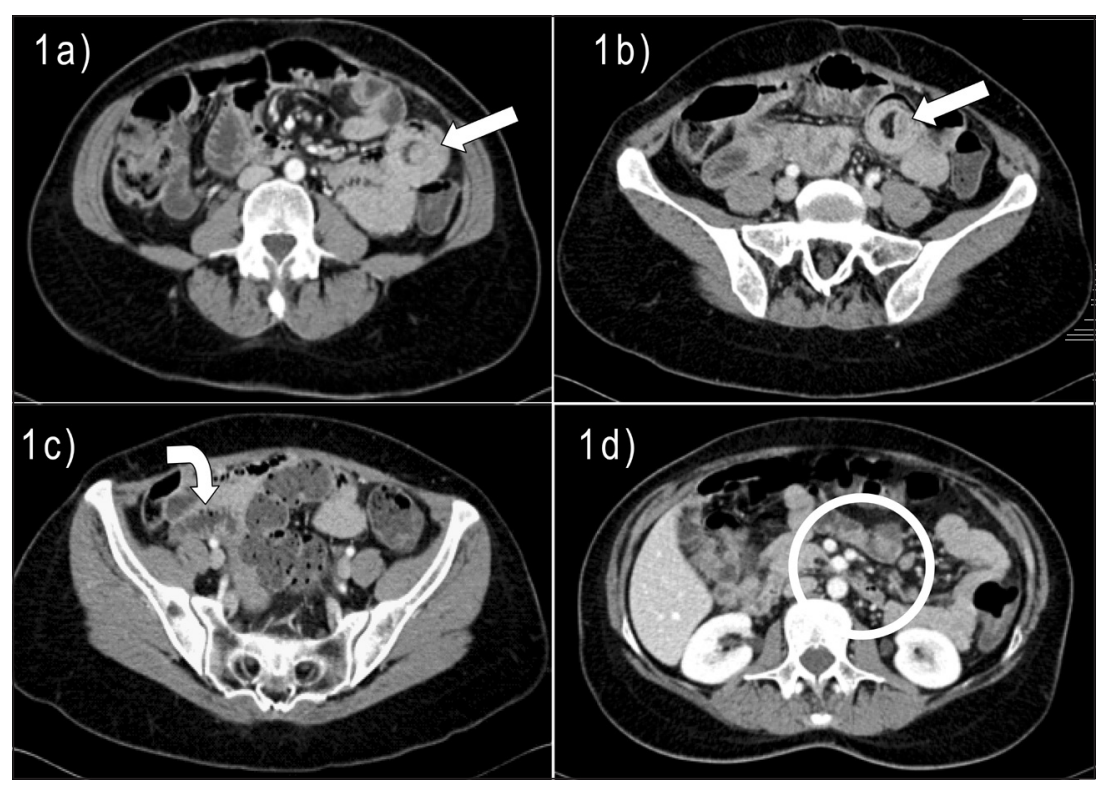

Figura 1. Alteraciones en TC de abdomen y pelvis (cortes axiales). 1a) y $1 \mathrm{~b}$ ): Signo de la crescente (grasa mesentérica invaginada) que confirma el diagnóstico de invaginación intestinal (flechas rectas). 1c): Aumento del número de pliegues a nivel de íleon (flecha curva). 1d): Pequeñas adenopatías mesentéricas (en círculo). 

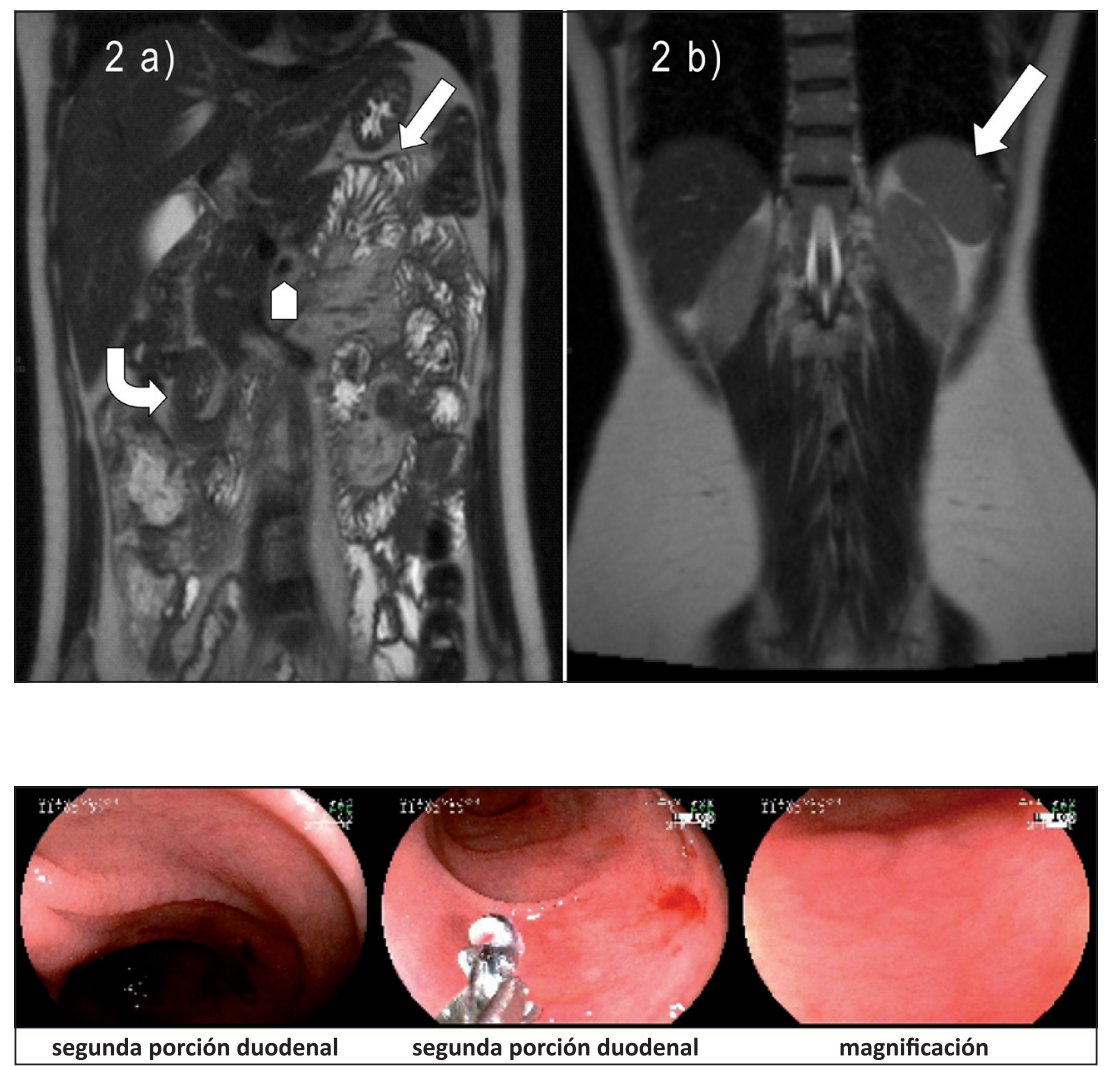

Figura 2. Alteraciones en la resonancia magnética de hígado (T2 HASTE coronal). 2a): Invaginación de asa de yeyuno en flanco derecho (flecha recta), asas de yeyuno distendidas en flanco izquierdo (flecha curva) y arteria mesentérica superior prominente (punta de flecha). 2b): Hipoesplenia (flecha recta).

Figura 3. Alteraciones a nivel de duodeno en la endoscopia digestiva alta. Pérdida difusa de las vellosidades intestinales con aspecto fenestrado y en ciertas áreas tipo empedrado a nivel de la segunda y tercera poción duodenal. llazgos: invaginación transitoria de un asa de yeyuno en flanco derecho, asas distendidas a nivel de yeyuno, aumento significativo en el número de pliegues a nivel de las asas de íleon, linfonodos mesentéricos prominentes, origen aberrante de la arteria mesentérica superior e hipoesplenia. Estos signos radiológicos plantearon la posibilidad de una EC (Figura 2A-C). Se solicitaron exámenes serológicos (anticuerpo antiendomisio positivo y anticuerpo antitransglutaminasa $67 \mathrm{U} / \mathrm{ml}$; valor positivo $>25 \mathrm{U} / \mathrm{ml}$ ). La endoscopia (Figura 3) e histología (Figura 4) confirmaron el diagnóstico de EC. La paciente inició tratamiento con restricción de gluten evolucionando hasta la fecha en buenas condiciones generales sin referir síntomas digestivos en la actualidad.

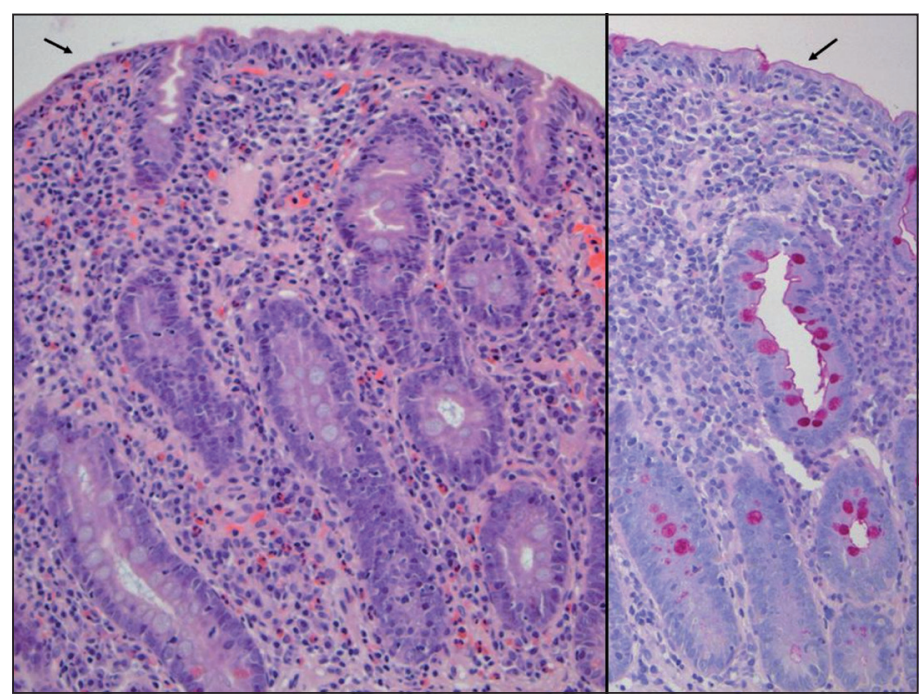

Figura 4. Alteraciones histológicas a nivel de duodeno. Marcada atrofia vellositaria con hipertrofia de las criptas e intensa linfocitosis intraepitelial (Marsh III-C). 


\section{Discusión}

La EC, una de las enfermedades gastrointestinales inmunes más frecuentes en Europa y Estados Unidos de Norteamérica, ha sido generalmente caracterizada como una enfermedad que suele presentarse en la infancia luego de la introducción del gluten. Sin embargo, estudios han demostrado que puede afectar a cualquier grupo etario y determinar una multiplicidad de síntomas clínicos ${ }^{3,15}$, situación que explica probablemente la demora en su diagnóstico. Las manifestaciones clínicas de la EC incluyen no sólo síntomas gastrointestinales inespecíficos como diarrea crónica, baja de peso o distensión abdominal, sino también síntomas secundarios a compromiso óseo (osteoporosis), dermatológico (alopecia, dermatitis herpetiforme), neurológico (ataxia, epilepsia) y reproductor (infertilidad) entre otros ${ }^{2,15}$.

La EC no diagnosticada puede ocasionalmente presentarse como un cuadro de urgencia ${ }^{16}$. Sanders y cols. mostraron que 3\% de los pacientes con dolor abdominal inespecífico presentaron el diagnóstico de EC. Sin embargo, este porcentaje aumentó a $10,5 \%$ al momento de considerar a aquellos pacientes con dolor abdominal no quirúrgico ${ }^{17}$. En pacientes con EC, el dolor abdominal puede ser causado por linfoma intestinal, adenocarcinoma intestinal, necrosis, perforación o invaginación intestinal. Distintas series y casos clínicos han demostrado la asociación entre EC e invaginación intestinal idiopática ${ }^{10-14}$. Recientemente, un estudio encontró que 11 de los 14 pacientes con EC e invaginación intestinal presentaron dolor abdominal al momento del diagnóstico ${ }^{14}$. Quizás el aporte más importante de nuestro artículo es confirmar que la invaginación intestinal puede ser una manifestación intestinal de la EC. Los mecanismos propuestos para el desarrollo de la invaginación intestinal en pacientes con EC, serían la presencia de inflamación a nivel de la mucosa asociado a la disfunción reversible del sistema nervioso extrínseco, lo que produciría una disminución de la motilidad a nivel del intestino delgado ${ }^{18,19}$. Estudios han sugerido que la presencia de invaginación intestinal se asocia a una mayor severidad de la atrofia de las vellosidades, pacientes con EC refractaria tipo II y linfoma células T asociado a enteropatía ${ }^{14,20}$. Tanto en el caso clínico publicado por Dodds y cols, como el nuestro, el paciente presentó un diagnóstico de EC Marsh
III-C ${ }^{13}$. Por otra parte, aunque la mayoría de los casos de invaginación intestinal en pacientes con EC no presentan un punto de origen, el adenocarcinoma y linfoma intestinal son complicaciones que pueden ocurrir en estos pacientes y deben ser consideradas al momento de determinar las posibles causas de la invaginación ${ }^{15,21}$.

La observación que la invaginación intestinal puede estar presente en pacientes con EC, evaluados por ecotomografía abdominal, TC de abdomen y pelvis o resonancia ${ }^{22-23}$, sugiere que estos pacientes pueden sufrir invaginaciones intestinales de manera frecuente y transitoria. Tomei y cols mostraron que en un grupo de 28 pacientes con EC, las alteraciones radiológicas más frecuentes en el TC de abdomen y pelvis fueron: alteración en el patrón de los pliegues intestinales $82 \%$, dilatación intestinal $75 \%$, aumento del líquido intestinal $64 \%$, linfoadenopatías $43 \%$, prominencia de los vasos mesentéricos $25 \%$, engrosamiento de la pared intestinal $21 \%$, invaginación intestinal $21 \%$, aumento del contenido intestinal de aire $14 \%$ y ascitis $7 \%{ }^{24}$. Estas mismas alteraciones han sido descritas utilizando la resonancia magnética como método de evaluación radiológica ${ }^{24}$. En nuestro paciente se encontraron seis de estos criterios.

Finalmente, dos puntos nos parecen importantes. Primero, que la EC debe ser considerada al momento de evaluar a pacientes con osteoporosis $^{25}$, sobre todo si esta se presenta a una edad temprana, como es el caso de nuestra paciente. Segundo, que la EC debe ser considerada en el diagnóstico diferencial del síndrome de intestino irritable ${ }^{26}$. Ambas situaciones estaban presentes en nuestro paciente antes que presentara el cuadro de invaginación intestinal y se diagnosticara la EC.

En conclusión, nosotros confirmamos que el dolor abdominal agudo puede ser una manifestación clínica en pacientes con EC. La invaginación idiopática, sobre todo si está asociada a otras alteraciones radiológicas, debe plantear la posibilidad de una EC. Teniendo esta manifestación clínica en mente será posible disminuir el número de paciente con EC no diagnosticada.

\section{Referencias}

1. Green PH, Cellier C. Celiac disease. N Engl J Med 2007; 357: 1731-43.

2. Herrera MJ, Hermosos M, Quera R. Enfermedad celíaca 
y su patogenía. Rev Med Chile 2009; 137: 1617-26.

3. Rewers M. Epidemiology of celiac disease: what are the prevalence, incidence, and progression of celiac disease? Gastroenterology 2005; 128: 547-51.

4. Lohi S, Mustalahti K, Kaukinen K, Laurila K, Collin $\mathrm{P}$, Rissanen $\mathrm{H}$, et al. Increasing prevalence of celiac disease over time. Aliment Pharmacol Ther 2007; 26: 1217-25.

5. Huppertz HI, Soriano-Gabarro M, Grimprel E, Franco E, Mezner Z, Desselberger U, et al. Intussusception among Young in Europe. Pediatr Infect J 2006; 25: S22-9.

6. Marinis A, Yiallourou A, Samanides L, Dafnios N, Anastasopoulos G, Vassiliou I, et al. Intussusception of the bowel in adults. A review. World J Gastroenterol 2009; 15: 407-11.

7. Lvoff N, Breiman RS, Coakley FV, Lu Y, Warren RS. Distinguishing features of self-limiting adult small-bowel intussusceptions indentified at CT. Radiology 2003; 227: 68-72.

8. Kim YH, Blake MA, Harisinghani MG, Archer-Arroyo K, Hahn PF, Pitman MB, et al. Adult intestinal intussusceptions: CT appearances and identification of a causative lead point. Radiographics 2006; 26: 733-4.

9. Agha FP. Intussusception in adults. AJR Am J Roentgenol 1986; 146: 527-31.

10. Martínez G, Israel NR, White JJ. Celiac disease presenting as entero-enteral intussusception. Pediatric Surg Int 2001; 17: 68-70.

11. Fishman DS, Chumpitazi BP, Ngo PD, Kim Hb, Lightdale JR. Small bowel intussusception in celiac disease: Revisiting a classic association. J Pediatr Gastroenterol Nutr 2010; 50: 237.

12. Makay O, Kazimi M, Doğanavşargil B, Osmanoğlu N, Yilmaz M. Acute abdomen in adult celiac disease: As intestinal intussusception case. Turk J Gastroenterol 2007; 18: 103-6.

13. Dodds BF, Aguancha SI, Santamarina RM, Vega SJ. Obstrucción intestinal por intususcepción como primera manifestación de enfermedad celíaca en adulto. Rev Med Chile 2008; 136: 1179-82.

14. Gonda TA, Khan SU, Cheng J, Lewis SK, Rubin M, Green $\mathrm{PH}$. Association of intussusception and celiac disease in adults. Dig Dis Sci 2009 Diciembre 24. Epub ahead of print.
15. Tack GI, Verbbek WH, Schreurs MW, Mulder CJ. The spectrum of celiac disease: epidemiology, clinical aspects and treatment. Nat Rev Gastroenterol Hepatol 2010; 7: 204-13.

16. Sanders DS, Hurlstone DP. Do patients with unrecognized coeliac disease presents as an emergency?. Eur J Emerg Med 2005; 12: 303-5.

17. Sanders DS, Hopper AD, Amzy IA, Rahman N, Hurlstone DP, Leeds JS, et al. Association of adult celiac disease with surgical abdominal pain: a case-control study in patients referred to secondary care. Ann Surg 2005; 242: 201-7.

18. Bassotti G, Villanacci V, Mazzocchi A, Mariano M, Incardona P, Clerici C, et al. Antroduodenojejunal motor activity in untreated and treated celiac disease patients. J Gastroenterol Hepatol 2008; 23: e23-8.

19. Bassotti G, Castellucci G, Betti C, Fusaro C, Cavalletti ML, Bertotto A, et al. Abnormal gastrointestinal motility in patients with celiac sprue. Dig Dis Sci 1994; 39: 1497 54.

20. Garrido A, Luque A, Vázquez A, Hernández JM, Alcántara F, Márquez JL. Primary small bowel neoplasms as a complication of celiac disease. Gastroenterol Hepatol 2009; 32: 618-21.

21. Mallant M, Hadithi M, Al-Toma AB, Kater M, Jacobs M, Manoliu R, et al. Abdominal computed tomography in refractory coeliac disease and enteropathy associated T-cell lymphoma. World J Gastroenterol 2007: 13; 1696700.

22. Bartusek D, Valek V, Husty J, Uteseny J. Small bowel ultrasound in patients with celiac disease. Retrospective study. Eur J Rad 2007; 63: 302-6.

23. Tomei E, Diacinti D, Marini M, Mastropasqua M, Di Tola M, Sabbatella L, et al. Abdominal CT findings may suggest coeliac disease. Dig Liver Dis 2005; 37: 402-6.

24. Tomei E, Semelka R, Braga L, Laghi A, Paolantonino P, Marini M, et al. Adult celiac disease: what is the role of MRI?. J Magn Reson Imaging 2006; 24: 625-9.

25. Duerksen DR, Leslie WD. Positive celiac disease serology and reduced bone mineral density in adult women. Can J Gastroenterol 2010; 24: 103-7.

26. Jadallah KA, Khader YS. Celiac disease in patients with presumed irritable bowel syndrome: a case-finding study. World J Gastroenterol 2009;15: 5321-5. 